Warum überhaupt Visionen?

Michael Krausz

\title{
Why bother with Visions?
}

Vielfach erscheint schon der therapeutische Alltag als belastend und angefüllt mit Mühseligkeiten; welchen Platz hat da noch die Zukunft? Oftmals erwischt sich jeder klinisch Tätige bei der Frage, in welche Zukunft denn das Wertesystem steuert, und man rekapituliert etwas sentimental und in der Regel nostalgisch überzeichnend die guten alten Zeiten. Das Interessante an dieser Art von Diskussionen ist, dass sie offensichtlich zeitlos sind. Sie haben zu nahezu jedem Zeitpunkt die Blicke verklärt und Veränderungen blockiert.

Dabei sind gerade die schwierigen Zeiten ein zusätzlicher Grund, über das Unmögliche nachzudenken, Lösungen jenseits ausgetretener Pfade zu suchen und über Veränderungen nachzudenken.

Vielleicht ist es hilfreich, angesichts so einer Kontroverse zu resümieren: Was ist schwierig in der jetzigen Entwicklungsphase des Suchthilfesystems?

\section{Mehr Möglichkeiten/neue Erfolgsparameter}

Das therapeutische Spektrum hat sich verändert. Die Behandlungsparadigmen und Erfolgskriterien sind im Umbruch. Es gibt nicht nur die Dichotomie Abstinenz oder Abhängigkeit. Der Umgang mit der Sucht beginnt, sich der wirklichen Wirklichkeit mehr anzunähern. Dies stellt insbesondere für die Beschäftigten im Hilfesystem eine enorme Herausforderung dar, denn das, was über Jahrzehnte als Messlatte der Effektivität und Ziel jeder Behandlung angesehen wurde, wird infrage gestellt, das, was als therapeutisches Prinzip angesehen wurde, wird weder von den Klienten und ihren Familien noch von der Wissenschaft als sinnvoller Umgang akzeptiert. Das gesamte Hilfesystem könnte also am Beginn eines Transformationsprozesses stehen. Es ist verunsichernd und schwierig.

\section{Das Verhältnis von Notwendigkeiten und Ressourcen}

Vieles von dem, was unbestreitbar notwendig wäre, um früh zu intervenieren und komplex Gestörten bzw. Abhängigen adäquat zu helfen, kann nur bedingt oder gar nicht finanziert werden. Bei der jetzigen Verteilung und Menge an Ressourcen liegt der schwarze Peter beim Hilfesystem: einerseits für die Klienten zu kämpfen, für ausreichend Ressourcen zu sorgen, andererseits die wachsende Einschränkung des Gesundheitswesens zu begrenzen und im Rahmen einer gesellschaftlichen Auseinandersetzung darüber Position zu gewinnen.

\section{Stigmatisierung von Suchtkranken}

Suchtkranke sind in unserer Gesellschaft immer noch in höherem Ausmaß stigmatisiert als andere Patientengruppen. Ihnen wird trotz wissenschaftlicher Evidenz vielfach wirkungsvolle Behandlung vorenthalten. Die Akzeptanz von Sucht als Krankheit ist immer noch begrenzt und kann bei Konkurrenzsituationen leicht umkippen. Das färbt auch auf die Therapeutinnen und Therapeuten ab. Sie werden oftmals ebenso in die Ecke gestellt. Suchtarbeit und Suchtforschung sind nach wie vor kaum Karriereschneisen in unserer Gesellschaft.

Wahrscheinlich gäbe es noch einige Gründe mehr zum Klagen. Aber schon diese machen deutlich, wie widersprüchlich die Situation ist, und dass aktuelle Probleme auch den Kern von neu-

Institutsangaben

Zentrum für Interdisziplinäre Suchtforschung der Universität Hamburg

Korrespondenzadresse

Prof. Dr. M. Krausz c/o Zentrum für Psychosoziale Medizin, Psychiatrie und Psychotherapie im UKE .

Martinistraße $52 \cdot 20246$ Hamburg

Bibliografie

Suchttherapie 2003; 4: 54-55 @ Georg Thieme Verlag Stuttgart · New York · ISSN 1439-9903 
en zukunftsträchtigen Ansätzen in sich haben. Gerade, wenn es schwierig wird, ist es notwendig, sich einen Blick von weiter weg zu genehmigen, um den Überblick zurückzugewinnen, vielleicht auch den Blick auf das Wesentliche. Dieser ist insbesondere bei jungen Mitarbeiterinnen und Mitarbeitern in größerem Ausmaß vorhanden und für sie ein wesentlicher Grund gewesen, sich im therapeutischen Beruf oder gar im Suchthilfesystem zu engagieren, bei Entwicklungen und Veränderungen helfen zu können, mit den schwierigsten Entwicklungen zu neuen Ansatzpunkten und Horizonten zu finden. Das ist auch der Grund für die Beiträge zu Paradigmen zwischen Alltag und Visionen gewesen. Ein bewusst gesetzter Akzent in der Entwicklungsdebatte. Warum sind also gelegentliche oder auch kontinuierliche Visionen gesundheitsfördernd, motivierend und vor allem orientierend?

Sie helfen bei der Rückbesinnung auf Werte und legen Motive in der eigenen oder auch kollektiven Entwicklung frei, die manchmal im Rahmen des Alltages zu verschwinden drohen.

Sie stärken die Motive, die es begründen, mehr zu tun, als seine Stunden abzuleisten, und mit Engagement in individuelle Schicksale einzugreifen, Entwicklungsperspektiven auf persönli- cher Ebene zu entwickeln, aber auch auf systemischer Ebene nicht aus dem Auge zu verlieren!

Sie sind die notwendige Grundlage für die Weiterentwicklung der alltäglichen Behandlungsparadigmen, Behandlungsphilosophien als Grundlage des eigentlichen therapeutischen Alltages. In diesem Sinne ist dieses Heft ein Versprechen, in dieser Richtung genauso viel Energie und Kreativität zu entfalten wie im Umgang mit klinisch therapeutischen Erfahrungen und ihrer Weiterentwicklung.

Es ist kein Wunder, dass die, die sich diese Fähigkeit immer wieder erkämpft haben, zu den besonderen Menschen in unserem Fach und in der Medizin insgesamt gehören: solche, die weit jenseits des offiziellen Pensionsalters noch eine Botschaft vertreten, nicht arbeiten, weil sie müssen, sondern weil sie der Suchtforschung, der Therapie, aber vor allem den Menschen innerlich verpflichtet sind, wie Ambros Uchtenhagen, dem Impulsgeber zu diesen Gedanken.

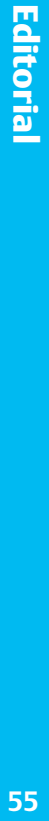

